\title{
Cólico renal: Revisión de la literatura y evidencia científica
}

\author{
S. Esquena, F. Millán Rodríguez, F.M. Sánchez-Martín, F. Rousaud Barón, \\ F. Marchant, H. Villavicencio Mavrich
}

Servicio de Urología. Fundació Puigvert. Barcelona.

Actas Urol Esp 2006; 30 (3): 268-280

\section{RESUMEN}

\section{CÓLICO RENAL: REVISIÓN DE LA LITERATURA Y EVIDENCIA CIENTÍFICA}

En los últimos años el diagnóstico y el tratamiento del cólico renal agudo han cambiado con la introducción de nuevos métodos diagnósticos que permiten determinar con muy alta fiabilidad la causa obstructiva, pudiendo establecer la estrategia terapéutica más adecuada. Por otro lado, la aparición de múltiples estudios dirigidos al tratamiento ha permitido clasificar los fármacos más efectivos para tratar la crisis aguda. El objetivo de esta revisión es la puesta al día del cólico renal haciendo énfasis en aquellos aspectos diagnóstico-terapéuticos que se han modificado con los estudios recientes, y que han permitido romper clásicos conceptos que se ha demostrado que carecían de evidencia científica.

Palabras clave: Cólico. Riñón. Uréter. Litiasis. Obstrucción.

\section{ABSTRACT}

RENAL COLIC: REVISION OF LITERATURE AND SCIENTIFIC EVIDENCE

In recent years diagnosis and treatment of acute renal colic have changed thanks to the introduction of new radiological procedures that allow with high reliability to establish obstructive cause and a better treatment. In the other hand, there are multiple clinical studies that show the most effective treatments for acute crisis. The aim of this revision is to update the diagnostic and therapeutic aspects of renal colic which were modified recently, and allowed to break some classic concepts without scientific evidence.

Keywords: Colic. Kidney. Uréter. Stone. Obstruction.

$\mathrm{E}_{\mathrm{i}}^{1}$ 1 cólico renal (CR) es una urgencia urológica frecuente, que comporta una de las formas más angustiantes de dolor en el ser humano, por lo que requiere de un rápido diagnóstico y tratamiento. Más de un $12 \%$ de la población sufrirá un CR durante su vida, siendo la tasa de recurrencia alrededor de un $50 \%{ }^{1}$. Es causado por una obstrucción ureteral aguda, parcial o completa, que en la gran mayoría de casos suele ser debida a un cálculo, provocando una distensión aguda del sistema colector.

\section{EPIDEMIOLOGÍA}

La frecuencia es mayor por la mañana $\mathrm{y}$ durante las estaciones calurosas ${ }^{2}$, por la menor producción urinaria nocturna y por situaciones con mayores pérdidas insensibles ${ }^{2}$, como sudoración intensa. Estas circunstancias aumentarian significativamente la concentración urinaria, actuando como un claro factor predisponente para desarrollar un ataque, que afectaría más a hombres de edad avanzada ${ }^{3}$. Estudios epidemiológicos norteamericanos muestran que los hombres caucasianos tienen la mayor incidencia, seguidos en orden por las mujeres caucasianas, mujeres y hombres de raza negra ${ }^{4,5}$. En estudios epidemiológicos que incluyen población hispánica norteamericana no se observan diferencias comparando con caucásicos y por el género, pero se detecta un mayor número de intervenciones 
urológicas por litiasis sintomáticas en las mujeres hispánicas ${ }^{6}$. El $25 \%$ de los pacientes que presentan cólicos renales recurrentes tienen historia familiar de urolitiasis $^{7}$, y con antecedentes de historia familiar el riesgo litiásico se multiplica por tres ${ }^{8}$. Al revisar estudios epidemiológicos de nuestra área, sólo encontramos referencias muy locales, por tanto, sería necesario un estudio amplio estatal epidemiológico que actualmente no existe en la literatura.

\section{FISIOPATOLOGÍA}

El brusco aumento de presión intraluminal debido a la obstrucción ureteral aguda se extiende desde les terminaciones nerviosas nociceptoras (quimiorreceptores y mecanorreceptores) localizadas en la submucosa y en la lámina propia de la cápsula renal peripiélica (responsables del dolor renal), la pelvis renal (responsables del dolor reno-ureteral), y en menor densidad, en el uréter proximal ${ }^{9}$. Además, la musculatura lisa de la pared ureteral se contrae intentando expulsar la obstrucción, y si no lo consigue, se espasmodiza. Una contracción prolongada isotónica conduce a una mayor producción de ácido láctico que irritará las fibras lentas tipo A (mielinizadas) y las rápidas tipo $\mathrm{C}$ (no mielinizadas). Estos impulsos nerviosos generados viajan hasta los segmentos medulares D1 1 - L2, llegando al sistema nervioso central, donde son especificadas por localización, carácter e intensidad, hecho que potenciará la crisis $^{10}$. Parece ser que la irritación local de la mucosa juega un papel menor ${ }^{10,11}$. La distribución por dermatomas del dolor renal es el resultado de una convergencia somato-visceral de la información neural recibida desde la médula espinal, de manera que el dolor se puede percibir en cada órgano que comparte inervación con el tracto urinario. Esto explica la típica irradiación del dolor desde las fibras espinales a las fibras aferentes renales y ureterales, y las fibras sensitivas desde la piel, correspondientes a aquellas zonas inervadas por los nervios genitofemoral, ilioinguinal e iliohipogástrico ${ }^{12}$. El resto de síntomas viscerales que con frecuencia se asocian al cólico renal (náuseas, vómitos, taquicardia, disminución del peristaltismo intestinal) son debidos a las múltiples conexiones existentes entre los plexos renal, celíaco y mesentérico que llegan a la médula espinal al mismo nivel provocando irritación vecinal ${ }^{12}$.

Se han descrito 3 fases sucesivas en la obstrucción ureteral aguda: la primera, que puede durar de 0 a 1,5 horas, donde aumentan el flujo sanguíneo renal (FSR) y la presión ureteral (PU), en la segunda, de 1,5 a 5 horas, cae el FSR mientras la PU continua aumentando, y finalmente, encontramos una tercera fase, más allá de las 5 horas, donde FSR y PU disminuyen conjuntamente $^{12}$. El aumento inicial del FSR es debido a una vasodilatación preglomerular, que muchos estudios demuestran que es secundaria a una aumento de producción local de eicosanoides, principalmente prostaglandina E2 (PGE2) y prostaciclina (PGI2) ${ }^{13}$, y donde el aumento de óxido nítrico (NO) también tendría un papel relevante ${ }^{13-15}$. Por otro lado, la PGE2 inhibe la secreción de hormona antidiurética $(\mathrm{ADH})$ durante la obstrucción aguda, hecho que incrementa más la PU, por el aumento del flujo urinario. Posteriormente, el FSR y la PU disminuyen en un mecanismo de autodefensa por la subsiguiente vasoconstricción preglomerular que aumentará las resistencias intrarenales, siendo una reacción establecida por diferentes mediadores, angiotensina II, tromboxano A2, ADH y endotelinas ${ }^{1,15}$. La disminución de la PU es secundaria a una disminución muy importante del filtrado glomerular pero que siempre persiste dentro de unos mínimos en un fenómeno que se explicaría porque la orina acumulada en la pelvis renal podría escapar por reflujos pielovenosos y pielolinfáticos, entre otros. Todo este proceso explicaría, en parte, la observación clínica de una mejoría espontánea de la intensidad del dolor algunas horas después de su inicio, en la mayoría de pacientes ${ }^{14,15}$.

Es importante destacar que el dolor de cólico renal no es causado directamente por contracciones espasmódicas del uréter obstruido, y que por tanto, el uso de fármacos espasmolíticos tendrán escasa utilidad, y además, podrían ser contraproducentes al inhibir el peristaltismo fisiológico ureteral dificultando y retardando la eliminación de la causa obstructiva.

En algunos casos, una prolongada e intensa hiperpresión dentro del sistema colector podría implicar una anulación muy severa del filtrado glomerular, pero antes de que esto suceda puede 
producirse una rotura de la vía urinaria, a nivel de los fórnix caliciales, con extravasación importante de orina a nivel perirenal. Esta puede ser reabsorbida por los sistemas venosos y linfáticos del espacio intersticial, siendo otro mecanismo de defensa para disminuir la tensión intraluminal, y las presiones piélica y ureteral, y que incluso se inicia antes de la rotura de la vía. Afortunadamente, el riesgo de fracaso renal definitivo no se establece hasta varias semanas después de haberse establecido una obstrucción completa con anulación máxima del filtrado glomerular, sin que la literatura haya establecido con precisión el tiempo necesario para provocar lesiones renales irreversibles (de 2 a 6 semanas según los autores y en modelos experimentales animales) ${ }^{16-18}$.

\section{ETIOLOGÍA}

En la gran mayoría de los pacientes, hasta un 90\%, el cólico es debido a una obstrucción ureteral aguda por una litiasis. En un 5-10\%, es debido a alteraciones de la vía urinaria no litiásicas, como, síndrome de la unión pieloureteral, presencia de coágulos por tumoraciones renales, atonía ureteral por pielonefritis, necrosis papilar o procesos neoplásicos uroteliales.

El resto de pacientes con cólico renal pueden presentar una obstrucción ureteral extrínseca secundaria a otros procesos, como enfermedades intestinales (apendicitis, diverticulitis, enfermedad de Crohn), ginecológicas, retroperitoneales, vasculares (aneurismas aortoilíacos, uréter retrocavo), oncológicas y hematológicas o complicaciones postquirúrgicas ${ }^{19}$.

\section{DIAGNÓSTICO}

\section{CLÍNICA}

El dolor de cólico renal suele ser de inicio súbito, unilateral y muy intenso. Se localiza en la fosa lumbar y en el ángulo costovertebral resiguiendo el margen inferior de la $12^{\mathrm{a}}$ costilla. Se irradia característicamente siguiendo el trayecto ureteral antero-descendente hasta vejiga, genitales externos, e incluso, cara interna del muslo. A veces, el paciente refiere que el dolor se inicia en las zonas donde típicamente se irradia, presentando posteriormente el dolor lumbar típico. El paciente presenta gran agitación, buscando una posición antiálgica imposible de encontrar $\mathrm{y}$ característicamente, sujetándose la fosa renal. Es posible que el médico determine la posición de un cálculo ureteral por la zona de irradiación. Si el cálculo está alojado en la porción superior del uréter, el dolor se irradia al testículo, ya que la inervación de este órgano es similar a la del riñón y la porción superior del uréter. Cuando el cálculo se encuentra en la porción media del uréter derecho, el dolor se irradia al punto de McBurney, pudiendo simular una apendicitis. En el lado izquierdo se puede parecer a una diverticulitis o a otras enfermedades del colon descendente o sigma. Conforme el cálculo se acerca a la vejiga, se produce inflamación y edema del orificio ureteral, apareciendo síntomas de irritabilidad vesical (polaquiuria y tenesmo).

La sintomatología no urinaria más frecuentemente asociada suele ser digestiva, en forma de náuseas, vómitos y constipación por íleo reflejo.

La fiebre no forma parte de la sintomatología del cálculo renal no complicado, aunque puede existir febrícula. Al explorar al paciente, palparemos un abdomen blando, sin signos de peritonismo, y con cierto timpanismo por el íleo reflejo. La suave percusión renal del lado afecto será positiva, incluso con contractura de la musculatura lumbar. En el hombre, el testículo con frecuencia está retraído y elevado, pero no es doloroso a la palpación. En la mujer, el examen pélvico será normal.

En la anamnesis, además de les características clínicas propias, para orientar más el cuadro, podemos buscar antecedentes personales y familiares de cólico renal, hematuria de esfuerzo, expulsión espontánea de pequeñas litiasis, etc., así como antecedentes familiares de litiasis urinaria, que se observa en el 3-10\% de los casos. Además, puede haber factores predisponentes de urolitiasis como inmovilización prolongada, enfermedades con manifestaciones óseas (hipertiroidismo, Paget, sarcoidosis, mieloma, etc.), y enfermedades digestivas (rectocolitis hemorrágica, ileitis, resecciones de ileon, enfermedades inflamatorias intestinales). También, ciertos tratamientos pueden ser responsables de cálculos metabólicos (quimioterapia oncológica de litiasis úrica; vitamina $\mathrm{D}$, calcio $\mathrm{y}$ furosemida de litiasis cálcica; inhibidores de la anhidrasa carbónica de litiasis de fosfato cálcico) o cálculos de origen medicamentoso (triamterene, sulfamidas, nitrofurantoina, indinavir) ${ }^{19,20}$. 


\section{EXPLORACIONES COMPLEMENTARIAS}

\section{Análisis urinario y sanguíneo}

En la mayoría de cólicos renales existirá hematuria macro o microscópica, aunque en un 9-33\% de casos está ausente ${ }^{21,22}$. Esta ausencia de hematuria no estaría relacionada con el grado de obstrucción ni con el tamaño y localización de la litiasis, como demuestran diferentes autores ${ }^{23,24}$.

La realización de un tira reactiva de orina será una prueba barata y suficiente en el estudio inicial de un cólico renal en el Servicio de Urgencias, ya que se demuestra más sensible que el recuento microscópico de hematíes en orina, hecho explicable por la hemólisis y las limitaciones propias de la microscopía como técnica, aunque algunos estudios refieren una eficacia diagnóstica similar de ambos métodos ${ }^{25,26}$.

Parece que el periodo comprendido entre el inicio del dolor y la recogida de orina presentaría un impacto significativo en el diagnóstico de hematuria con tira reactiva, observando que la ausencia de hematuria se produciría mayoritariamente en los días 3 y 4 después del inicio del dolor, coincidiendo con la hemostasia fisiológica secundaria a la impactación de la litiasis ${ }^{27}$.

Es también frecuente la presencia de leucocitos, aunque no exista infección asociada, consecuencia de la propia reacción inflamatoria, siendo la tira reactiva muy sensible y específica para detectar leucocituria, y muy específica, pero con una sensibilidad del $50 \%$ para nitritos, que equivaldría a hallar bacterias.

El análisis sanguíneo no será necesario en la evaluación de un cólico renal simple, siendo de utilidad ante la sospecha clínica de cólico renal complicado (presencia de fiebre, oligo-anuria en un monorreno, obstrucción bilateral) donde los parámetros más relevantes a valorar serán el recuento leucocitario, si existe desviación a la izquierda de la fórmula, y la función renal, así como otros parámetros de sepsis como las pruebas de coagulación o la PCR.

\section{EXPLORACIONES RADIOLÓGICAS}

Les exploraciones radiológicas permitirán apurar el diagnóstico, aunque no suelen cambiar la actitud terapéutica en Urgencias delante de un cólico renal simple con claro diagnóstico clínico, pero serán imprescindibles en la evaluación de un cólico renal complicado:
Radiografia simple de abdomen: es el estudio de imagen más rápido y sencillo en los pacientes con cólico renal. Esta prueba detecta los cálculos ureterales con una sensibilidad que varía del 45 al 59\%, por lo que la su utilidad es limitada. La superposición del intestino y de los huesos oculta algunos cálculos, y los flebolitos (perlas venosas calcificadas, redondas, múltiples, con frecuencia muy excéntricas, con menor densidad central, y muy frecuentes en los espacios paravesicales), se pueden confundir con litiasis. Además, la radiografía simple no detecta cálculos radiotransparentes (ácido úrico puro), litiasis menores de $2 \mathrm{~mm}$ o microlitiasis (popularmente llamadas "arenilla"), ni la causa obstructiva cuando no es litiásica (hasta un 10\% de casos), y la presencia de una calcificación abdominal no es un signo seguro de que esté localizada en el aparato urinario. Permite ver cálculos a partir de $2 \mathrm{~mm}$ si son de calcio y a partir de 3-4 $\mathrm{mm}$ si son de densidad más baja (estruvita o cistina). Además, podemos observar, una ligera escoliosis de concavidad hacia el lado afecto, un discreto aumento de tamaño de la silueta del riñón afectado y posibles imágenes radioopacas sugestivas de cálculos a nivel renal o ureteral ${ }^{19}$.

Ecografia abdominal: es un método incruento, rápido, portátil, repetible, relativamente barato y que no usa radiaciones ionizantes ni material de contraste. Nos aporta información sobre el estado de la vía por encima de la obstrucción, informando del grado de ectasia pielocalicial del riñón afecto (se considera que una separación mayor de $5 \mathrm{~mm}$ de los ecos en el seno renal constituye un signo indirecto de obstrucción), pudiendo diagnosticar, además, litiasis radiotransparentes no visibles en la radiografía simple, o procesos tumorales que comprometan el vaciado ureteral. El uso del Doppler permitirá estudiar el índice de resistencia renal (parámetro que permite detectar obstrucción con alta sensibilidad y especifici$\mathrm{dad}^{28}$, observándose una disminución del índice del riñón obstruido respecto al contralateral, y que incluso permitiría diferenciar, si el proceso es de instauración aguda o crónica) y la evaluación de los jets ureterales (presencia y simetría de los eyaculados de ambos uréteres). El análisis de estos parámetros permitiría detectar obs- 
trucción renal aguda y al ofrecer resultados funcionales, podría permitir establecer un valor predictivo del paso de la litiasis, pudiendo reemplazar a la UIV $^{29}$ en el cólico renal litiásico, sobre todo en contraindicaciones de la UIV, como el embarazo ${ }^{30}$.

Como deben transcurrir horas para que se desarrolle una pielocaliectasia manifiesta después de una obstrucción súbita e incluso completa, no resulta sorprendente que la ecografía pase por alto el $20-30 \%$ de les obstrucciones agudas causadas por un cálculo ureteral. Además, se pueden establecer diagnósticos falsamente positivos de obstrucción en pacientes con pielonefritis, ectasias crónicas residuales, reflujo vesicoureteral, dilatación residual después de aliviar la obstrucción o sobredistensión de la vejiga, sobretodo en mujeres. También debe destacarse la disminución de su eficacia diagnóstica en uréter medio y distal, siendo útil para valorar causas obstructivas en el uréter yuxtavesical con la vejiga repleccionada. Por tanto, resulta de gran utilidad la combinación de la radiografía simple de abdomen y de la ecografía, con demostración de los cálculos y la pielocaliectasia, en el diagnóstico de cólico renal agudo. Es muy importante destacar que la mayoría de cólicos renales presentan un mayor o menor grado de ectasia en la ecografía, y por tanto, no debe considerarse como un signo de complicación per se, además, el grado de ectasia no tiene una relación directa con la intensidad del dolor, ya que este síntoma estaría más relacionado con la velocidad de instauración de la obstrucción, cuanto más rápida, más intensidad. De esta forma, su uso en el cólico renal simple no es obligado pero sí cuando el cuadro es complicado o con dudas diagnósticas de la causa obstructiva. También es importante recordar que se han asociado contracciones peristálticas irregulares de amplitud inferior con infecciones del tracto urinario superior provocadas por endotoxinas producidas por enterobacterias ${ }^{31}$, y esta situación podría mostrar cierta ectasia de la vía por ecografía en una unidad renal que realmente no estaría obstruida.

Urografia intravenosa: durante mucho tiempo se ha considerado el método diagnóstico de elección para estudiar a los pacientes con cólico renal. Está al alcance de todos los hospitales, es fiable, barato y relativamente inocuo. Los hallazgos clásicos en la obstrucción renal aguda consisten en, retraso en la aparición del nefrograma, que adquiere una densidad cada vez mayor en las imágenes posteriores, retraso en la aparición del contraste en el sistema pielocalicial, dilatación ureteral proximal en la zona de la obstrucción y posible identificación de la causa. Tiene el inconveniente de las reacciones alérgicas debidas al contraste, que han disminuido con los contrastes de baja osmolaridad. Muchos centros siguen considerando la UIV como el método de referencia para diagnosticar el cólico renal agudo. No obstante, con la introducción de la ecografía Doppler con medición del índice de resistencia renal y la TAC helicoidal sin contraste con reconstrucción tridimensional, la UIV tenderá a usarse cada vez $\operatorname{menos}^{32}$.

TC helicoidal sin contraste: varios estudios recientes han confirmado a esta exploración como una modalidad precisa para la evaluación del cólico renal, con una sensibilidad de hasta el $98 \%$ y una especificidad de hasta un $100 \%{ }^{32,33}$. No requiere de contraste, siendo especialmente atractiva en pacientes alérgicos o con insuficiencia renal previa. Puede visualizar pequeños cálculos, incluidos los radiotransparentes, y si la litiasis es suficientemente pequeña para no ser identificada, se observan múltiples signos indirectos como dilatación ureteral y renal, trazos de líneas de grasa perirenal, líquido perirenal y el signo del anillo en las partes blandas (visualización de un círculo de tejido blando que envuelve al cálculo, que representa la pared edematosa del uréter, permitiendo diferenciar los cálculos de los flebolitos). Es un procedimiento rápido de tan solo 5 minutos, y que en algunos sistemas sanitarios se ha determinado que no cuesta más que la UIV ${ }^{33}$. Además, cuando no existe litiasis identifica con exactitud otras alteraciones urinarias y no urinarias. Las principales desventajas de esta prueba, en comparación con la UIV, son que no evalúa la función renal, y no en todos los centros se dispone de servicio de TC, sobretodo durante 24 horas, y legalmente se requiere la presencia de un radiólogo para la cumplimentación de la exploración. 


\section{DIAGNÓSTICO DIFERENCIAL}

El cólico renal, sobre todo en sus formas atípicas, puede simular numerosas afecciones:

- Renales: pielonefritis aguda, embolia-infarto renal.

- Genitales: torsión de cordón espermático, torsión de ovario, embarazo extrauterino, salpingitis.

- Digestivas: apendicitis aguda, oclusión intestinal, diverticulitis, pancreatitis aguda.

- Vasculares: disección o rotura de aneurisma de aorta abdominal.

- Neurológicas: lumbo-ciatalgia, neuralgia lumbo-abdominal o ilio-lumbar, tabes.

En la práctica clínica será muy importante establecer el diagnóstico diferencial entre cólico renal febril o pielonefritis aguda obstructiva y pielonefritis aguda simple (Tabla 1).

\section{TRATAMIENTO}

El manejo conservador se considera la primera línea de tratamiento del cólico renal simple ${ }^{34}$ ya que dos tercios de las litiasis ureterales son expulsadas espontáneamente en las 4 semanas siguientes al inicio de los síntomas ${ }^{35}$. Una litiasis ureteral que no ha sido expulsada después de 1 a 2 meses es altamente improbable que se expulse espontáneamente ${ }^{35}$. Los objetivos del tratamiento son establecer un buen control del dolor, y conservar al máximo la función renal suprimiendo o aliviando los efectos de la obstrucción ureteral ${ }^{1,34}$.
Existen múltiples estrategias a diferentes niveles fisiopatológicos para el tratamiento del cólico renal.

\section{HIDRATACIÓN, FLUIDOS Y DIURÉTICOS}

Parecería razonable pensar que a un paciente con un cólico renal agudo, aquellas medidas encaminadas a aumentar la producción urinaria, como el uso de diuréticos o el aumento de volumen de fluidos administrados, podrían, teóricamente, favorecer el paso de la litiasis al aumentar la presión hidrostática dentro del uréter, disminuyendo la duración e intensidad de los síntomas. Estudios de metanálisis no han podido establecer evidencia científica en este tema, y si añadimos el riesgo potencial de rotura de la vía y fracaso renal, podríamos concluir que estas medidas no serían recomendables $^{36}$.

Por otro lado, parece clara la recomendación de evitar una excesiva sobrehidratación durante la fase aguda del cólico renal, que provocaría una exacerbación de los síntomas y un riesgo potencial de rotura de la vía, sin evidencia que disminuyan los tiempos de expulsión ${ }^{36}$.

\section{CALOR LOCAL}

Existen múltiples estudios que muestran la eficacia del calor local para disminuir el dolor y la angustia en traumatismos menores ${ }^{37,38}$, incluso en dolores de origen cardíaco ${ }^{39}$ y dolores menstruales en mujeres jóvenes ${ }^{40}$.

\section{Tabla 1}

Diagnóstico diferencial entre pielonefritis aguda obstructiva y pielonefritis aguda simple

\section{Cólico renal febril o pielonefritis aguda obstructiva}

- Suele iniciarse en forma de dolor lumbar de tipo cólico.

- Sintomatología vegetativa concomitante muy frecuente.

- Sd. Irritativo miccional (no estranguria) coincidiendo o posterior al dolor lumbar por probable litiasis yuxtavesical.

- Febrícula/fiebre después de horas/días de iniciarse el dolor lumbar.

- Antecedentes pers./famil. de lts. Renal.

- Con frecuencia la rx abdomen evidencia litiasis.

- La ecografia suele mostrar ectasia de la vía.

- Es una urgencia urológica y requiere drenaje de la vía (cateterismo ureteral vs nefrostomía percutánea)

\section{Pielonefritis aguda simple}

- Suele debutar en forma de síndrome miccional en las horas o días previos.

- Se continua con dolor lumbar sordo y fiebre/afectación estado general precoz.

- La rx no evidencia litiasis y tira reactiva de orina habitualmente piúrica.

- Antecedentes personales de itus previas.

- Ausencia de ectasia en la ecografía.

- El tratamiento es estrictamente médico (antibiótico) 
El mecanismo de actuación analgésico se explicaría porque el calor local actuaría distorsionando los estímulos de los nociceptores periféricos y su procesamiento en la médula dorsal, causando una reducción de la actividad simpática provocada por el dolor cólico, y consiguiendo una disminución en la percepción de este dolor ${ }^{40}$. Algunos estudios demuestran que la aplicación de calor local en el cólico renal es una medida efectiva y fácil de aliviar del dolor, y útil como tratamiento complementario en el cuadro agudo $^{40}$.

\section{TRATAMIENTO FARMACOLÓGICO \\ Anticolinérgicos}

Los fármacos anticolinérgicos como la N-butilbromuro de hioscina (Buscapina ${ }^{\circledR}$ ) se han utilizado clásicamente como analgésicos en el cólico renal al inducir una relajación de la musculatura lisa con disminución del espasmo ureteral, que ha sido la explicación fisiopatológica clásica del dolor. Actualmente es suficientemente conocido que el mecanismo fisiopatológico del dolor es la distensión de la cápsula renal por la obstrucción, siendo el espasmo ureteral resultante una respuesta que contribuye de forma menor al cuadro. A pesar de todo, todavía está muy extendido el uso de estos fármacos como tratamiento adyuvante de AINEs y opiáceos en la crisis aguda. Aunque existen estudios que demuestran que el uso de antimuscarínicos disminuye el dolor en comparación con placebo, ningún estudio ha demostrado que sean tan eficaces como opiáceos, AINEs $u$ otros analgésicos cuando son usados como fármaco único ${ }^{41-43}$. Además, existen estudios que muestran que la adición de un antimuscarínico a los AINEs u opiáceos no aporta beneficios analgésicos, ni disminuye la sintomatología vegetativa acompañante ${ }^{44,45}$. Con todos estos resultados y disponiendo en nuestro medio de una batería de fármacos más eficaces y más dirigidos al origen fisiopatológico del dolor, no está justificado el uso de fármacos antimuscarínicos en el tratamiento del cólico renal. Además, sería de interés valorar en estudios posteriores si la adición de antimuscarínicos al tratamiento, al disminuir el peristaltismo ureteral, retardaría el progreso ureteral de la causa obstructiva, dificultando y aumentando el tiempo de expulsión.

\section{Antiinflamatorios no esteroideos (AINEs)}

Múltiples estudios muestran que los AINEs proporcionan un significativo alivio del dolor en el cólico renal ${ }^{46,47}$. Aparte de su potente efecto analgésico y antiinflamatorio, tienen el beneficio teórico de actuar directamente sobre la causa principal del dolor, inhibiendo la síntesis de prostaglandinas, y por tanto, disminuyendo el FSR, reduciendo la producción urinaria y la presión intraluminal. Estudios de metanálisis han demostrado que son, como mínimo, tan efectivos como los opiáceos en el tratamiento del cólico renal ${ }^{48}$. Los efectos secundarios más frecuentes están bien definidos, hemorragia gastrointestinal e insuficiencia renal, aunque con una correcta protección gástrica durante el tratamiento, y en pacientes sin insuficiencia renal previa, no se ha detectado ningún efecto adverso grave en amplios estudios de metanálisis $^{49,54}$. Sólo deberían evitarse en pacientes alérgicos o con insuficiencia renal previa, para no agravarla.

Existe una amplia variedad de fármacos, dosis y vías de administración, así naproxeno y diclofenac aportan una potente analgesia con baja incidencia de efectos adversos, el ibuprofeno es el que tiene el menor riesgo de efectos gastrointestinales, y la indometacina tiene un efecto muy potente pero con alta incidencia de efectos $\operatorname{adversos}^{50}$. El diclofenac y la indometacina por vía oral o rectal muestran alta eficacia, con reducción de los reingresos hospitalarios por dolor y el diclofenac tiene menos efectos adversos que por vía parenteral ${ }^{50}$. El uso de diclofenac vía rectal puede ser de utilidad en pacientes con náuseas o vómitos que impidan la tolerancia oral, con comparable efectividad analgésica y escasos efectos adversos ${ }^{51}$. Por otro lado, varios estudios demuestran que el diclofenac podría aumentar la reabsorción tubular, retardando la excreción de contraste renal, sobreestimando el retraso de eliminación en pruebas de contraste realizadas en fase aguda ${ }^{52,53}$.

En estudios de metanálisis donde se realizan comparaciones de AINEs frente a opiáceos para el tratamiento del cólico renal, se concluye que los AINEs consiguen una mayor reducción en las puntuaciones del dolor y es menos probable que requieran de analgesia adicional a corto término con me- 
nos efectos secundarios que los opiáceos ${ }^{49,54}$. Por tanto, se convendría que cuando se requiera de un bolus único de analgesia en la fase aguda del cólico renal, un AINE sería el fármaco de elección ${ }^{54}$ en lugar de un opiáceo, y también se recomendaría su uso, siempre que no estuviera contraindicado, en el tratamiento posterior de mantenimiento, a la espera de futuros estudios que definan cuales son los fármacos y las vías de administración idóneas.

\section{Opiáceos}

Está ampliamente demostrado en la literatura que los opiáceos proporcionan una franca disminución del dolor en el cólico renal agudo ${ }^{49}$. Tienen la ventaja de su bajo coste, facilidad de ajustar dosis, alta potencia y rapidez de actuación, con el efecto negativo de la dependencia que pueden provocar. Por otro lado, a pesar de su potencia analgésica, no actúan sobre el origen fisiopatológico del dolor. Se han demostrado de eficacia prácticamente similar a los AINEs en el cólico renal agudo ${ }^{48}$, con ligera ventaja de los AINEs, aunque se desconoce su dosis óptima para conseguir una analgesia adecuada.

Se observa mayor frecuencia de efectos adversos en los pacientes tratados con opiáceos, donde los vómitos se asociaron claramente, sobre todo con el uso de petidina. Por todo esto podemos establecer que los opiáceos son fármacos muy eficaces en el tratamiento del cólico renal agudo, que están indicados como tratamiento complementario de los AINEs o cuando éstos están contraindicados o se requiere de un ajuste de dosis, y se recomendaría no usar la petidina por la alta tasa de vómitos asociada.

\section{Dipironas}

Las dipironas son analgésicos no opiáceos de uso muy frecuente en algunos países como España y Brasil, pero muy poco utilizados en países anglosajones, aduciendo el grave riesgo potencial de agranulocitosis ${ }^{55}$. Se han demostrado como una analgesia efectiva para el tratamiento del cólico renal agudo, en dosis única o como tratamiento complementario de AINEs y opiáceos ${ }^{55,56}$. Estudios de metanálisis muestran que administrados por vía intramuscular son menos efectivos que el diclofenac $75 \mathrm{mg}$, y que su uso junto con espasmolíticos no mejoran su eficacia. Además, se observa que vía endovenosa son más efectivos que intramusculares y en la mayoría de las series publicadas no se ha descrito ningún caso de agranulocitosis, siendo los efectos adversos más frecuentes descritos, somnolencia y sequedad de boca ${ }^{55}$. Por otro lado, algún estudio sugiere que en el cólico renal agudo el uso de $2 \mathrm{~g}$ de dipirona no estaría justificado ya que es igualmente efectiva una dosis de $1 \mathrm{~g}^{57}$.

\section{Otros analgésicos}

El uso de paracetamol en el cólico renal agudo está escasamente definido, pero no sería considerado un fármaco de elección inicial. Estaría indicado durante el embarazo o en contraindicación de los fármacos anteriormente descritos, con la ventaja de no presentar los efectos adversos del resto $^{58}$.

Existen algunos estudios que valoran la eficacia de la desmopresina intranasal en el cólico renal agudo aduciendo su facilidad de uso, efecto muy rápido y ausencia de efectos adversos, ofreciendo una buena eficacia analgésica, tanto sola como administrada junto con diclofenac, incrementando su efecto ${ }^{59,60}$. Este fármaco actuaría reduciendo intensamente la presión ureteral por su potente efecto antidiurético, sin los efectos presores de la vasopresina, como se demuestra en modelos experimentales ${ }^{61}$. Por otro lado, favorecería la liberación de beta-endorfinas hipotalámicas, aunque esta teoría continua en discusión ${ }^{62}$.

A pesar que todavía existen escasos estudios sobre el uso de los inhibidores de la COX-2 para el cólico renal agudo, ya se ha establecido en estudios experimentales, que reducen la contractilidad ureteral, además de su potente efecto antiinflamatorio, evitando efectos adversos gástricos ${ }^{63}$. Actualmente sólo están disponibles por vía oral. Existen estudios comparativos realizados con rofecoxib, antes de su retirada del mercado, que mostraban una disminución de los requerimientos analgésicos de mórficos en cólico agudo de forma similar al diclofenac ${ }^{64}$. Por tanto, el potente efecto antiinflamatorio de los inhibidores de la COX-2, junto con su actuación directa sobre la fisiopatología del dolor y con escasos efectos adversos, permite que se muestren como 
fármacos potencialmente muy útiles a la espera de futuros estudios que mejoren la evidencia científica disponible actualmente, usando otros fármacos de la misma familia (celecoxib, etoricoxib, parecoxib).

\section{Fármacos no analgésicos: Antieméticos,}

Hipnóticos

En una crisis de cólico renal agudo serán muy útiles diferentes fármacos para controlar la sintomatología vegetativa acompañante. Los antieméticos, como la metoclopramida, ayudarán al control de las náuseas y vómitos causados por irritación vecinal de los plexos celíaco y mesentérico, y actuará como un procinético para evitar la disminución del peristaltismo que se puede producir en la fase aguda. Por otro lado, la agitación y la taquicardia causadas por el intenso dolor cólico pueden ser tratadas sintomáticamente con hipnóticos tipo benzodiazepinas.

Infiltración y bloqueo subcutáneo paravertebral.

La infiltración subcutánea de un anestésico local (lidocaína 2\% o bupivacaína 0,25\%) para provocar un bloqueo paravertebral en el tratamiento del cólico renal agudo es un método simple, barato, sin efectos adversos, pero con el inconveniente de su corta duración ${ }^{65}$. La punción se realizaría a nivel paravertebral entre D10 y L2, entre el ángulo escapular, las apófisis espinosas y la cresta ilíaca. Fue descrito inicialmente en la literatura médica soviética y es una práctica médica habitual en Rusia ${ }^{66}$. El mecanismo de actuación es incierto, pero se ha demostrado que la efectividad es similar al inyectar suero salino ${ }^{65}$. Algunos autores explican el fenómeno por la "teoría de la puerta" del dolor que consistiría en que la punción de los dermatomas correspondientes, inhibiría circuitos neurales medulares hasta un nivel central, bloqueando la transmisión de los nociceptores ${ }^{67}$.

\section{Litotricia extracorpórea por ondas de choque}

(LEOC) urgente

Una opción de tratamiento en el cólico renal agudo es la realización de una LEOC urgente para fragmentar la litiasis responsable, a las horas siguientes de la presentación del cuadro. Recientes estudios demuestran que es un proceso seguro y que ofrece un buen control del dolor con resolución de la obstrucción, disminuyendo la necesidad de hospitalización y el retorno a la actividad normal del paciente, en comparación con una LEOC diferida ${ }^{68,69}$. Los mejores resultados se obtienen en litiasis menores de $5 \mathrm{~mm}$ y situadas por debajo de los vasos ilíacos, a pesar que en litiasis de mayor tamaño y más proximales, los resultados también son mejores que difiriendo la $\mathrm{LEOC}^{70}$.

\section{TRATAMIENTOS EN SITUACIONES ESPECIALES}

Cólico renal por litiasis ureteral distal

En los últimos años encontramos múltiples estudios que postulan la utilización de un tratamiento combinado con un corticosteroide, habitualmente deflazacort, más un calcio-antagonista o un $\alpha$-bloqueante, para la litiasis ureteral distal. El uso de $\alpha$-bloqueantes se explicaría fisiopatológicamente por la mayor densidad de receptores $\alpha 1$ en el uréter distal, siendo menor en uréter medio y proximal, como se demuestra en estudios inmunohistoquímicos ${ }^{76}$, y los calcio-antagonistas actuarian como relajantes de la musculatura lisa ureteral. Los primeros estudios se realizaron usando un calcio-antagonista, habitualmente nifedipino ${ }^{71}$, y en estudios más recientes, se ha usado la tamsulosina ${ }^{72}$, con buenos resultados. Este tratamiento combinado estaría indicado en litiasis expulsables de uréter distal (por debajo de los vasos ilíacos), y permitiría aumentar significativamente la tasa de expulsión, disminuir el tiempo para expulsar, disminuyendo los requerimientos analgésicos y la necesidad de hospitalización e instrumentación ${ }^{71,72}$. Existe algún estudio comparativo entre calcio-antagonistas y tamsulosina, que muestra una clara ventaja usando tamsulosina para todos los parámetros definidos anteriormente ${ }^{73,74}$. También se han realizado comparaciones entre diferentes $\alpha$-bloqueantes, observando efectos similares al usar doxazosina, terazosina o tamsulosina ${ }^{75}$. El deflazacort es el corticosteroide usado en todos los estudios por su potencia antiedematosa y al no requerir de pauta descendente. De esta forma el uso de este tratamiento combinado en el cólico renal por litiasis uretral distal podría ser una opción recomendable. 


\section{Cólico renal post-LEOC}

El cólico renal simple que se presenta después de una sesión de LEOC se tratará de forma idéntica al que aparece espontáneamente, aunque debemos recordar el mayor riesgo de carretera litiásica posterior, siendo muy importante en este caso disponer de una exploración radiológica. Para restos litiásicos post-LEOC situados en el uréter distal, la pauta de tratamiento anteriormente descrita será útil. Por otro lado, algunos estudios demuestran la utilidad de la tamsulosina para reducir el número de cólicos renales e intensidad del dolor de los mismos, después de la LEOC y para favorecer la expulsión de los fragmentos restantes ${ }^{77}$, incluso en presencia de carreteras litiásicas $^{78}$.

\section{SITUACIONES ESPECIALES}

\section{Cólico renal en monorreno}

La actitud delante de un cólico en un paciente con un solo riñón es la misma que en un birreno, siempre y cuando el paciente no presente anuria, fiebre igual o superior $38^{\circ} \mathrm{C}$ o status cólico, ya que en estos casos lo trataremos como un cólico renal complicado ${ }^{78}$.

\section{Cólico renal durante el embarazo}

La urolitiasis es un problema infrecuente pero importante durante la gestación, y su incidencia es de 1 de 1.500 embarazos $^{20}$. Es más frecuente en multíparas y en el segundo, y sobre todo, en el tercer trimestre ${ }^{80,81}$. Su diagnóstico no siempre es fácil, y suele manifestarse por dolores en el flanco (90-100\%), o por hematuria macro o microscópica (75-100\%) ${ }^{80}$. Teniendo en cuenta las modificaciones anatómicas sufridas durante el embarazo, puede manifestarse por dolores abdominales (40-56\%), hecho que puede llevarnos al diagnóstico incorrecto de apendicitis, diverticulitis o desprendimiento precoz de placenta. Puede acompañarse de náuseas, vómitos o clínica irritativa miccional.

La mayor gravedad del cólico renal durante el embarazo es el riesgo de parto prematuro (40$67 \%)$. Aunque sólo en el primer trimestre es evidente el riesgo fetal si se usa radioscopia, se recomienda la ecografía como método diagnóstico más inocuo, que evidenciará pielocaliectasia de grado variable, con una sensibilidad mayor al
$90 \%$ para detectar una litiasis reno-ureteral ${ }^{81}$. La frecuente dilatación de las vías superiores inducida por el embarazo hace que la ecografía en escala de grises resulte inespecífica para detectar obstrucción, siendo de gran utilidad el modo Doppler. También es útil e inocua la resonancia magnética en secuencia rápida ${ }^{19,20}$.

El tratamiento del cólico renal en la gestación representa un problema importante porque puede provocar un parto prematuro y los procedimientos terapéuticos invasivos son potencialmente perjudiciales para el feto. Por tanto, se suelen recomendar tratamientos temporales conservadores. Los analgésicos más fiables son los opiáceos y el paracetamol, debiendo evitar los AINEs, por el riesgo de cierre prematuro del ductus arteriosus. En el 50-80\% de casos, el cálculo se expulsa espontáneamente ${ }^{80}$, pero cuando se asocia a sepsis o status cólico se requerirá tratamiento urgente. Dado que la LEOC está contraindicada, el cateterismo ureteral, la nefrostomía percutánea o incluso la ureteroscopia, en manos experimentadas, serán buenas opciones terapéuticas. Por otro lado, deberá realizarse profilaxis antibiótica de larga duración en pacientes embarazadas portadoras de tutor ureteral y que presenten bacteriuria ${ }^{81}$.

\section{Instrumentación/Desobstrucción quirúrgica de}

\section{la vía urinaria}

Deberá realizarse una desobstrucción de la vía urinaria delante de un cólico renal complicado, que podemos definir como aquel que se acompaña de los signos de gravedad siguientes:

- Fiebre $\left(\geq 38{ }^{\circ} \mathrm{C}\right)$ : expresa una infección del parénquima renal y de la orina por encima del obstáculo. El riesgo potencial es la sepsis de origen urinario, habitualmente provocada por bacilos gram-negativos, con gravísimas repercusiones sistémicas y alta mortalidad.

- Status Cólico: es la persistencia de las crisis de dolor durante más de 24 horas a pesar de mantener al paciente en tratamiento parenteral con múltiples fármacos cada 4 horas, siendo alguno de ellos, un opiáceo. Además del agotamiento del paciente a causa del dolor, existe un alto riesgo de rotura de la vía excretora. Si se apura correctamente el tratamiento farmacológico, suele ser una indicación muy poco habitual. 
- Anuria: ya sea por obstrucción bilateral o en riñón único, y con todas las complicaciones que implica una insuficiencia renal aguda.

A pesar que en la literatura urológica quedan establecidas estas indicaciones de instrumentación, actualmente todavía no existen estudios sobre cual es la mejor forma de desobstrucción para facilitar el tratamiento posterior de la litiasis, qué tipo de derivación aporta mayor calidad de vida al paciente hasta la resolución de la causa obstructiva, cuáles son las indicaciones precisas de cateterismo ureteral con catéter recto o con doble jota, así como otras muchas cuestiones que se plantean en la actitud a tomar delante de un cólico renal complicado, estando a la espera de futuros estudios para poder establecer evidencia científica sobre este tema.

\section{REFERENCIAS}

1. Shokeir AA. Renal colic: pathophysiology, diagnosis and treatment. Eur Urol. 2001;39(3):241-249.

2. Boari B, Manfredini R. Circadian rhythm and renal colic. Recenti Prog Med. 2003;94(5):191-193.

3. Chauhan V, Eskin B, Allegra JR, Cochrane DG. Effect of season, age, and gender on renal colic incidence. Am J Emerg Med. 2004;22(7):560-563.

4. Soucie JM, Thun MJ, Coates RJ, McClellan W, Austin H. Demographic and geographic variability of kidney stones in the United States. Kidney Int. 1994;46(3):893-899.

5. Sarmina I, Spirnak JP, Resnick MI. Urinary lithiasis in the black population: an epidemiological study and review of the literature. J Urol. 1987;138(1):14-17.

6. Dall'era JE, Kim F, Chandhoke PS. Gender Differences among Hispanics and Caucasians in symptomatic presentation of kidney and ureteral stones. J Endourol. 2005; 19(3):283-286.

7. Ljunghall S, Danielson BG, Fellstrom B, Holmgren K, Johansson G, Wikstrom B. Family history of renal stones in recurrent stone patients. Br J Urol. 1985;57(4):370-374.

8. Curhan GC, Willett WC, Rimm EB, Stampfer MJ. Family history and risk of kidney stones. J Am Soc Nephrol. 1997;8(10):1568-1573.

9. Moody TE, Vaughn ED Jr, Gillenwater JY. Relationship between renal blood flow and ureteral pressure during 18 hours of total unilateral uretheral occlusion. Implications for changing sites of increased renal resistance. Invest Urol. 1975;13(3):246-251.

10. Klahr S. New insights into the consequences and mechanisms of renal impairment in obstructive nephropathy. Am J Kidney Dis. 1991;18(6):689-699.

11. Cervero F, Sann H. Mechanically evoked responses of afferent fibres innervating the guinea-pig's ureter: an in vitro study. J Physiol. 1989;412:245-266.

12. Travaglini F, Bartoletti R, Gacci M, Rizzo M. Pathophysiology of reno-ureteral colic.Urol Int. 2004; 72 Suppl 1:20-23.

13. Schulman CC. Electron microscopy of the human ureteric innervation. Br J Urol. 1974;46(6):609-623.
14. Lanzone JA, Gulmi FA, Chou SY, Mooppan UM, Kim H. Renal hemodynamics in acute unilateral ureteral obstruction: contribution of endothelium-derived relaxing factor. $\mathrm{J}$ Urol. 1995;153(6):2055-2059.

15. Shokeir AA. Renal colic: new concepts related to pathophysiology, diagnosis and treatment. Curr Opin Urol. 2002;12(4):263-269.

16. Vela Navarrete R. El riñón dilatado, 2001. Editorial Mason.

17. Vela Navarrete R, García-Fisac JM. Complete ureteral obstruction: delayed manometric response and its physiopathologic significance Arch Esp Urol. 1983;36(6):383-390.

18. Vaughan ED Jr, Gillenwater JY. Recovery following complete chronic unilateral ureteral occlusion: functional, radiographic and pathologic alterations. J Urol. 1971;106 (1): 27-35.

19. Lechevallier E, Dussol B, Bretheau D. Enciclopedia MédicoQuirúrgica de Urología, 2001.

20. Walsh, Retik, Vaughan, Wein. Campbell's Urology. 8th ed. 2002.

21. Press SM, Smith AD. Incidence of negative hematuria in patients with acute urinary lithiasis presenting to the emergency room with flank pain. Urology, 1995;45:753-757.

22. Stewart DP, Kowalski R, Wong P, Krome R. Microscopic hematuria and calculus-related ureteral obstruction. J Emerg Med 1990;8:693-695.

23. Bove P, Kaplan D, Dalymple N, Rosenfield AT, Verga M, Anderson K, et al. Reexamining the value of hematuria testing in patients with acute flank pain. J Urol, 1999;162: 685-687.

24. Li J, Kennedy D, Levine M, Kumar A, Mullen J. Absent hematuria and expensive computerized tomography case characteristics of emergency urolithiasis. J Urol, 2001; 165:782-784.

25. Kiel DP, Moskowitz MA. The urinalysis: a critical appraisal. Med Clin North Am, 1987;71:607-624.

26. Vaughan ED Jr, Wyker AW Jr. Effect of osmolality on the evaluation of microscopic hematuria. J Urol. 1971;105(5): 709-711.

27. Kobayashi T, Nishizawa K, Mitsumori K, Ogura K. Impact of date of onset on the absence of hematuria in patients with acute renal colic. J Urol. 2003;170(4 Pt 1):1093-1096.

28. Geavlete P, Georgescu D, Cauni V, Nita G. Value of duplex Doppler ultrasonography in renal colic. Eur Urol. 2002;41 (1):71-78.

29. Kmetec A, Peskar-Babnik D, Buturovic-Ponikvar J. Timedependent changes of resistive index in acute renal obstruction during nonsteroidal drug administration. BJU Int. 2002;89(9):847-850.

30. Shokeir AA, Mahran MR, Abdulmaaboud M. Renal colic in pregnant women: role of renal resistive index. Urology. 2000;55(3):344-347.

31. King WW, Cox CE. Bacterial inhibition of ureteral smooth muscle contractility. I. The effect of common urinary pathogens and endotoxin in an in vitro system. J Urol. 1972; 108(5):700-705.

32. Shokeir AA, Abdulmaaboud M. Prospective comparison of nonenhanced helical computerized tomography and Doppler ultrasonography for the diagnosis of renal colic. J Urol. 2001;165(4):1082-1084.

33. Yilmaz S, Sindel T, Arslan G, Ozkaynak C, Karaali K, Kabaalioglu A, Luleci E. Renal colic: comparison of spiral CT, US and IVU in the detection of ureteral calculi. Eur Radiol. 1998;8(2):212-217. 
34. Hubner WA, Irby P, Stoller ML. Natural history and current concepts for the treatment of small ureteral calculi. Eur Urol. 1993;24(2):172-176.

35. Miller OF, Kane CJ. Time to stone passage for observed ureteral calculi: a guide for patient education. J Urol. 1999;162(3 Pt 1):688-90; discussion 690-691.

36. Rozanski TA, Edmondson JM. Fluids and diuretics for acute ureteric colic.Cochrane Database Syst Rev. 2005; 20;(3):CD004926.

37. Nuhr M, Hoerauf K, Bertalanffy A, Bertalanffy P, Frickey N, Gore C. et al. Active warming during emergency transport relieves acute low back pain. Spine. 2004 15; 29(14):14991503.

38. Kober A, Scheck T, Fulesdi B, Lieba F, Vlach W, Friedman A. et al. Effectiveness of resistive heating compared with passive warming in treating hypothermia associated with minor trauma: a randomized trial.Mayo Clin Proc. 2001; 76(4):369-375.

39. Hartman $\mathrm{CH}$. Response of anginal pain to hand warming. A clinical note. Biofeedback Self Regul. 1979;4(4):355-357.

40. Kober A, Dobrovits M, Djavan B, Marberger M, Barker R, et al. Local active warming: an effective treatment for pain, anxiety and nausea caused by renal colic. J Urol. 2003; 170(3):741-744.

41. Jones JB, Dula DJ. The efficacy of sublingual hyoscyamine sulfate and intravenous ketorolac tromethamine in the relief of ureteral colic. Am J Emerg Med. 1998;16(6):557559.

42. Stankov G, Schmieder G, Zerle G, Schinzel S, Brune K. Double-blind study with dipyrone versus tramadol and butylscopolamine in acute renal colic pain. World J Urol. 1994;12(3):155-161.

43. Benyajati C. Comparative study of Baralgan and hyoscine$\mathrm{N}$-methyl bromide in the treatment of intestinal and renal colicy pain. J Med Assoc Thai. 1986;69(10):569-573.

44. Jones JB, Giles BK, Brizendine EJ, Cordell WH. Sublingual hyoscyamine sulfate in combination with ketorolac tromethamine for ureteral colic: a randomized, double-blind, controlled trial. Ann Emerg Med. 2001;37(2): 141-146.

45. Holdgate A, Oh CM. Is there a role for antimuscarinics in renal colic? A randomized controlled trial. J Urol. 2005; 174(2):572-5; discussion 575.

46. Curry C, Kelly AM. Intravenous tenoxicam for the treatment of renal colic. N Z Med J. 1995;14;108(1001):229-230.

47. Smally AJ. Analgesia in renal colic. Ann Emerg Med. 1997;29(2):296.

48. Labrecque M, Dostaler LP, Rousselle R, Nguyen T, Poirier S. Efficacy of nonsteroidal anti-inflammatory drugs in the treatment of acute renal colic. A meta-analysis. Arch Intern Med. 1994;27;154(12):1381-1387.

49. Holdgate A, Pollock T. Nonsteroidal anti-inflammatory drugs (NSAIDs) versus opioids for acute renal colic. Cochrane Database Syst Rev. 2005;18;(2):CD004137.

50. Davenport K, Timoney AG, Keeley FX. Conventional and alternative methods for providing analgesia in renal colic. BJU Int. 2005;95(3):297-300.

51. Lee C, Gnanasegaram D, Maloba M. Best evidence topic report. Rectal or intravenous non-steroidal anti-inflammatory drugs in acute renal colic. Emerg Med J. 2005;22(9): 653-654.

52. Brough RJ, Lancashire MJ, Prince JR, Rose MR, Prescott MC, Payne SR, et al The effect of diclofenac (voltarol) and pethidine on ureteric peristalsis and the isotope renogram. Eur J Nucl Med. 1998;25(11):1520-1523.
53. Shokeir AA, Abdulmaaboud M, Farage Y, Mutabagani H. Resistive index in renal colic: the effect of nonsteroidal anti-inflammatory drugs. BJU Int. 1999;84(3):249-251.

54. Holdgate A, Pollock T. Systematic review of the relative efficacy of non-steroidal anti-inflammatory drugs and opioids in the treatment of acute renal colic. BMJ. 2004;12; 328(7453): 1401. Epub 2004 Jun 3.

55. Edwards JE, Meseguer F, Faura C, Moore RA, McQuay HJ. Single dose dipyrone for acute renal colic pain. Cochrane Database Syst Rev. 2002;(4): CD003867.

56. Stankov G, Schmieder G, Zerle G, Schinzel S, Brune K. Double-blind study with dipyrone versus tramadol and butylscopolamine in acute renal colic pain. World J Urol. 1994;12(3):155-161.

57. [Sin autores listados] Comparative study of the efficacy of dipyrone, diclofenac sodium and pethidine in acute renal colic. Collaborative Group of the Spanish Society of Clinical Pharmacology. Eur J Clin Pharmacol. 1991;40(6): 543-546.

58. Porena M, Guiggi P, Balestra A, Micheli C. Pain killers and antibacterial therapy for kidney colic and stones. Urol Int. 2004;72 Suppl 1:34-39.

59. Lopes T, Dias JS, Marcelino J, Varela J, Ribeiro S, Dias J. An assessment of the clinical efficacy of intranasal desmopressin spray in the treatment of renal colic. BJU Int. 2001;87(4):322-325.

60. Constantinides C, Kapralos V, Manousakas T, Mitropoulos D, Alamanis C, Dimopoulos C. Management of renal colic with intranasal desmopressin spray. Acta Urol Belg. 1998; 66(4): 1-3.

61. Moro U, De Stefani S, Crisci A, De Antoni P, Scott CA, Selli C. Evaluation of the effects of desmopressin in acute ureteral obstruction. Urol Int. 1999; 62(1):8-11.

62. el-Sherif AE, Salem M, Yahia H, al-Sharkawy WA, alSayrafi M. Treatment of renal colic by desmopressin intranasal spray and diclofenac sodium. J Urol. 1995;153(5): 1395-1398.

63. Nakada SY, Jerde TJ, Bjorling DE, Saban R. Selective cyclooxygenase- 2 inhibitors reduce ureteral contraction in vitro: a better alternative for renal colic? J Urol. 2000; 163(2): 607-612.

64. Engeler DS, Ackermann DK, Osterwalder JJ, Keel A, Schmid HP. A double-blind, placebo controlled comparison of the morphine sparing effect of oral rofecoxib and diclofenac for acute renal colic. J Urol. 2005;174(3):933-936.

65. Nikiforov S, Cronin AJ, Murray WB, Hall VE. Subcutaneous paravertebral block for renal colic. Anesthesiology. 2001;94(3):531-532.

66. Rossikhin VV. Effectiveness of M.N. Astvatsaturov's block in renal colic. Klin Khir. 1978;(2):74-75.

67. Wall PD, Melzak R Texbook of pain 3rt edition. New York, Churchill Livingstone, 1994;1191.

68. Kravchick S, Bunkin I, Stepnov E, Peled R, Agulansky L, Cytron S. Emergency extracorporeal shockwave lithotripsy for acute renal colic caused by upper urinary-tract stones. J Endourol. 2005;19(1):1-4.

69. Tligui M, El Khadime MR, Tchala K, Haab F, Traxer O, Gattegno B, et al. Emergency extracorporeal shock wave lithotripsy (ESWL) for obstructing ureteral stones. Eur Urol. 2003;43(5):552-555.

70. Tombal B, Mawlawi H, Feyaerts A, Wese FX, Opsomer R, Van Cangh PJ. Prospective randomized evaluation of emergency extracorporeal shock wave lithotripsy (ESWL) on the short-time outcome of symptomatic ureteral stones. Eur Urol. 2005;47(6):855-859. Epub 2005 Mar 17. 
71. Clayman RV. Effectiveness of nifedipine and deflazacort in the management of distal ureter stones. J Urol. 2002;167 (2 Pt 1):797-798.

72. Dellabella M, Milanese G, Muzzonigro G. Efficacy of tamsulosin in the medical management of juxtavesical ureteral stones. J Urol. 2003;170(6 Pt 1):2202-2205.

73. Dellabella M, Milanese G, Muzzonigro G. Randomized trial of the efficacy of tamsulosin, nifedipine and phloroglucinol in medical expulsive therapy for distal ureteral calculi. J Urol. 2005; 174(1):167-172.

74. Porpiglia F, Ghignone G, Fiori C, Fontana D, Scarpa RM. Nifedipine versus tamsulosin for the management of lower ureteral stones.J Urol. 2004;172(2):568-571.

75. Yilmaz E, Batislam E, Basar MM, Tuglu D, Ferhat M, Basar $H$. The comparison and efficacy of 3 different alpha1-adrenergic blockers for distal ureteral stones. J Urol. 2005;173 (6):2010-2012.

76. Sigala S, Dellabella M, Milanese G, Fornari S, Faccoli S, Palazzolo F, et al. Evidence for the presence of alpha1 adrenoceptor subtypes in the human ureter. Neurourol Urodyn. 2005;24(2):142-148.

77. Gravina GL, Costa AM, Ronchi P, Galatioto GP, Angelucci A, Castellani D, et al. Tamsulosin treatment increases clinical success rate of single extracorporeal shock wave lithotripsy of renal stones. Urology. 2005;66(1):24-28.
78. Resim S, Ekerbicer HC, Ciftci A. Role of tamsulosin in treatment of patients with steinstrasse developing after extracorporeal shock wave lithotripsy. Urology. 2005; 66(5): 945-948.

79. Ruiz Marcellan FJ, Ibarz Servio L, Ramon Dalmau M. Treatment of lithiasis in the patient with a solitary kidney. Eur Urol. 1988;15(1-2):13-17.

80. Stothers L, Lee LM. Renal colic in pregnancy. J Urol. 1992; 148(5):1383-1387.

81. Parulkar BG, Hopkins TB, Wollin MR, Howard PJ Jr, Lal A. Renal colic during pregnancy: a case for conservative treatment. J Urol. 1998;159(2):365-368.

Dr. Salvador Esquena

Servicio de Urología

Fundación Puigvert

C/Cartagena, 340-350

08025 Barcelona

e-mail: doc99@eresmas.com

sesquena@fundacio-puigvert.es

(Trabajo recibido el 16 de enero 2006) 